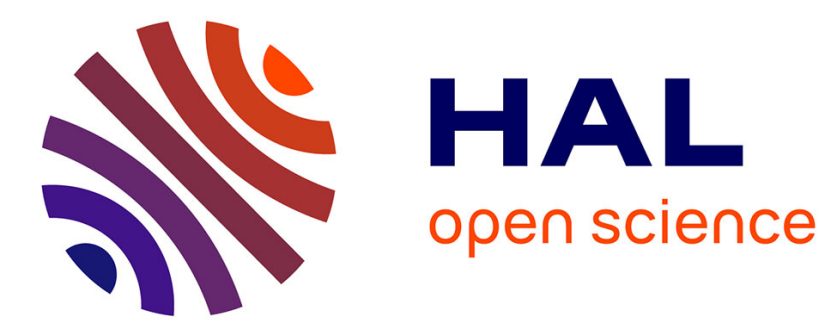

\title{
A gametheoretic approach for non-uniform pole shifting and pole homothety
}

Marc Jungers, Hisham Abou-Kandil, Eugênio Castelan, Edson Roberto de Pieri

\section{- To cite this version:}

Marc Jungers, Hisham Abou-Kandil, Eugênio Castelan, Edson Roberto de Pieri. A gametheoretic approach for non-uniform pole shifting and pole homothety. Automatica, 2013, 49 (1), pp.238-244. 10.1016/j.automatica.2012.09.021 . hal-00753147

\section{HAL Id: hal-00753147 https://hal.science/hal-00753147}

Submitted on 13 Jun 2013

HAL is a multi-disciplinary open access archive for the deposit and dissemination of scientific research documents, whether they are published or not. The documents may come from teaching and research institutions in France or abroad, or from public or private research centers.
L'archive ouverte pluridisciplinaire HAL, est destinée au dépôt et à la diffusion de documents scientifiques de niveau recherche, publiés ou non, émanant des établissements d'enseignement et de recherche français ou étrangers, des laboratoires publics ou privés. 


\title{
A Gametheoretic Approach for Non-Uniform Poles Shifting and Poles Homothety ${ }^{\star}$
}

\author{
Marc Jungers ${ }^{\mathrm{a}, \mathrm{b}}$, Hisham Abou-Kandil ${ }^{\mathrm{c}}$, Eugênio B. Castelan ${ }^{\mathrm{d}}$, \\ Edson R. De Pieri ${ }^{\mathrm{d}}$ \\ ${ }^{a}$ Université de Lorraine, CRAN, UMR 7039, 2 avenue de la forêt de Haye, Vandouvre-lès-Nancy Cedex, 54516, France \\ (email: marc.jungers@univ-lorraine.fr) \\ ${ }^{\mathrm{b}}$ CNRS, CRAN, UMR 7039, France \\ ${ }^{\mathrm{c}}$ SATIE, ENS Cachan, CNRS, 61 av du President Wilson, F-94230 Cachan, France (hisham@satie.ens-cachan.fr) \\ d $D A S-C T C-U F S C$ \\ P.O. Box 476, 88040-900 Florianópolis, SC, Brazil \\ (emails: [eugenio;edson]@das.ufsc.br)
}

\begin{abstract}
This paper investigates the problems of non-uniform pole shifting or pole homothety in multivariable linear systems by using a gametheoretic approach and more specifically a particular Nash strategy with an open-loop information structure for a game including a time preference rate in the quadratic criteria. Such a result is possible due to properties of non-symmetric Algebraic Riccati equations associated with an open-loop Nash strategy. Numerical examples allow to illustrate the efficiency of the proposed approach.
\end{abstract}

Key words: Pole Shifting, Pole Homothety, Game Theory, Nash Strategy, Coupled Algebraic Riccati Equations.

\section{Introduction}

Since the pioneering work of Rissanen [31] and Wonham [34], the problem of pole assignment for linear multivariable systems has obtained a great attention in the automatic control literature in both continuous and discrete time frameworks. The main result is called the pole assignment theorem which gave rise to different refinements and discussions [16,32]. A particular pole assignment in linear multivariable systems consists in shifting only the real part of the open-loop eigenvalues in continuous time domain or in applying an homothetic rate on them in discrete time domain. Such a technique, denoted pole shifting in the sequel for the sake of clarity, allows usually to obtain some desired time domain responses from a controlled process and has received a considerable attention [26,27,4,5,25].

One of the common features of all the aforementionned references is exploiting some interesting properties of the Algebraic Riccati Equation (ARE) associated with a linear-quadratic problem. This includes the mirror effect as in [26], the $\alpha$-stability approach for continuous time [5], the $\rho$-stability for discrete time [10] and the introduction of some parameters in the ARE as in $[27,4,6]$. Most of the proposed methods deal with a multiple step procedure where in each step, one real pole or a pair of complex poles are shifted to a desired location. An elegant iterative algorithm was also given in [25] where a special modification of the performance criteria associated with the use of

\footnotetext{
* A preliminary version of this paper, dealing only with the continuous time case was presented at the $18^{\text {th }}$ IFAC World Congress, Milano, Italia [21]. Corresponding author M. Jungers. Tel. + 33 (0) 383595704 . Fax + 33 (0) 383595644.
} 
the negative definite solution of the ARE allows pole shifting. The ARE approach is not the unique technique for poles placement or shifting: the LMI approach should be pointed out [11].

In a gametheoretic framework, it has been shown that introducing an exponential term in the criteria associated with each player leads to a global shift of poles for Stackelberg games (see [20] for continuous time and [19] for discrete time). Such modifications of the performance criteria in a differential or difference game are quite natural in studying decision problems in economy where the exponential term is associated with a specific discount rate for each player or decision maker [12]. However, in all cited references, no precise pole shifting procedure is proposed, only the observation that introducing discount rates in the criteria causes pole shifts of the dynamic or state matrix was made. In fact, the gametheoretic approach involves Coupled (Differential or) Algebraic Riccati Equations (CARE) belonging to the set of non symmetric algebraic Riccati equations, which are more difficult to handle and do not exhibit the nice poles symmetry properties of standard ARE. On the other hand, to avoid solving CARE, pole shifting is used as a central step in an inverse solution procedure to impose some time domain constraints [18].

In this paper, a single step, direct and simple method to operate non-uniform multiple pole shifting for continuous time and non-uniform multiple pole homothety for discrete time for linear multivariable systems are proposed. The non iterative algorithm is based on a special formulation of a linear-quadratic Nash differential or difference game with an open-loop information structure scheme. By exploiting some properties of the corresponding non symmetric CARE, a feedback gain matrix is easily determined such that all poles of the closed loop system have the desired locations. The possible existence of several solutions for the CARE leading to the same closed loop system will be used to provide several feedback gains associated with the desired pole shifting.

The paper is organized as follows. In Section 2, preliminary results on generalized Riccati equations are recalled. Section 3 introduces the differential gametheoretic framework and the Nash strategy with open-loop information structure. The solution of such a game problem via non symmetric CARE is presented in Section 4 . Section 5 settles the problem of non-uniform poles shifting and provides a solution by defining particular a Open-Loop Nash differential game. Sections 6 and 7 treat the case of discrete-time games for applying the non-uniform poles homothety. Numerical examples illustrate the efficiency and the simplicity of the obtained results in Sections 8 and 9 , before making some concluding remarks in Section 10.

Notations. Relative to a matrix $A \in \mathbb{R}^{m \times n}, A^{T}$ denotes its transpose. $I_{n}$ denotes the $n \times n$ identity matrix and $0_{n}$ the $n \times n$ null matrix. $\operatorname{Span}(M)$ is the vector space span generated by the columns of matrix $M$. For a square matrix $M, \lambda(M)$ denotes the spectrum of $M$. For a complex scalar or vector $z, \bar{z}$ is the conjugate of $z$. Finally, the symbol «•» in a matrix denotes a block matrix with no sense value.

\section{Preliminary results on generalized Riccati equations}

Let us consider a rectangular matrix variable $K(t) \in \mathbb{R}^{r n \times n}$, with $r$ and $n$ two integers. This section is related to solving the Generalized Differential Riccati Equation (GDRE) [1,14] (also called Rectangular one, or non-symmetric one) on the finite time interval $\left[t_{0}, t_{f}\right]$

$$
\left\{\begin{aligned}
\dot{K} & =-K \mathcal{N}_{11}+\mathcal{N}_{22} K+\mathcal{N}_{21}-K \mathcal{N}_{12} K \\
K_{f} & =K\left(t_{f}\right)
\end{aligned}\right.
$$

and the associated Generalized Algebraic Riccati Equation (GARE)

$$
0_{r n \times n}=-K \mathcal{N}_{11}+\mathcal{N}_{22} K+\mathcal{N}_{21}-K \mathcal{N}_{12} K
$$

where $\mathcal{N}_{11}, \mathcal{N}_{12}, \mathcal{N}_{21}$ and $\mathcal{N}_{22}$ have appropriated dimensions and may be gathered in a characteristic matrix

$$
\mathcal{N}=\left[\begin{array}{c:c}
\mathcal{N}_{11} & \mathcal{N}_{12} \\
\hdashline \mathcal{N}_{21} & \mathcal{N}_{22}
\end{array}\right] \in \mathbb{R}^{(r+1) n \times(r+1) n} .
$$

The solutions of the non-linear differential equation (1) can be obtained by a backward time integration on the 
time interval $\left[t_{0} ; t_{f}\right]$, from the final values (18). Nevertheless it is interesting to notice that the GDRE (1) may be linearized by the Radon's Lemma [30] involving the characteristic matrix $\mathcal{N}$ (see also [1, Theorem 3.1.1] or [13]).

Theorem 1 Let $K(t)$ be a solution of (1) on $\left[t_{0}, t_{f}\right]$. If $Q(t) \in \mathbb{R}^{n \times n}$ is a solution of the final value problem

$$
\dot{Q}(t)=\left(\mathcal{N}_{11}+\mathcal{N}_{12} K(t)\right) Q(t), \quad Q\left(t_{f}\right)=I_{n}
$$

and $P(t)=K(t) Q(t)$, then $Z(t)=\left(\begin{array}{c}Q(t) \\ P(t)\end{array}\right)$ is a solution of the associated linear system $\dot{Z}(t)=\mathcal{N} Z(t)$, with $Z\left(t_{f}\right)=\left(\begin{array}{c}I_{n} \\ K_{f}\end{array}\right)$. In the other side, if $Z(t)=\left(\begin{array}{c}Q(t) \\ P(t)\end{array}\right)$ is a solution of $\dot{Z}(t)=\mathcal{N} Z(t)$ such that $Q(t)$ is regular for $t \in\left[t_{0}, t_{f}\right]$, then $K(t)=P(t) Q^{-1}(t)$ is a solution of $(1)$.

Theorem 1 implies that the GDRE (1) may be solved by a backward time integration of the differential equation $\dot{Z}(t)=\mathcal{N} Z(t)$, on the time interval $\left[t_{0} ; t_{f}\right]$. When the time-horizon tends to infinity, that is by taking the limit $t_{f} \rightarrow+\infty$, the final value has no sense any more and the solution of the differential equations (1) become the ones of the GARE defined by (3). The solutions of the GARE are obtained via the invariant subspaces of matrix $\mathcal{N}$ (see $[1$, Theorem 6.2.2]).

Theorem 2 In one hand, $\mathcal{S}(K)=\operatorname{Span}\left(\begin{array}{c}I_{n} \\ K\end{array}\right)$ is an invariant subspace of $\mathcal{N}$ and the closed-loop matrix $A_{c l}=$ $\mathcal{N}_{11}+\mathcal{N}_{12} K$ is its matrix of restriction, i.e. $\mathcal{N}\left(\begin{array}{c}I_{n} \\ K\end{array}\right)=\left(\begin{array}{c}I_{n} \\ K\end{array}\right) A_{c l}$. In the other hand, if Span $\left(\begin{array}{c}X \\ Y\end{array}\right)$ is an invariant subspace of $\mathcal{N}$, with $X \in \mathbb{C}^{n \times n}$ invertible and $Y \in \mathbb{C}^{n r \times n}$, then

$$
K=Y X^{-1}
$$

is a solution of the GARE (3).

The possible spectra of the closed-loop matrix $A_{\mathrm{cl}}$ are not arbitrary, as shown in the following theorem.

Theorem 3 The eigenvalues of the closed-loop matrix $A_{\text {cl }}$ are among the eigenvalues of the matrix $\mathcal{N}$.

Proof The proof is obtained directly by writing $\left[\begin{array}{cc}I_{n} & 0_{n \times n r} \\ K & I_{n r \times n r}\end{array}\right]^{-1} \mathcal{N}\left[\begin{array}{cc}I_{n} & 0_{n \times n r} \\ K & I_{n r \times n r}\end{array}\right]=\left[\begin{array}{cc}A_{\mathrm{cl}} & \bullet \\ 0_{n r \times n} & \bullet\end{array}\right]$.

Due to theorems 2 and 3, it is possible to provide the following algorithm checking the solvability and giving the solutions of the GARE (3).

Algorithm 1 [1] Let a matrix $\mathcal{N}$ be given by (4).

Step 1: Select $n$ desired eigenvalues $\lambda_{i} \in \mathbb{C}(i=1, \cdots, n)$ among the ones of $\mathcal{N}$ to design the desired eigenspectrum $\Lambda_{\text {desired }}$ of $A_{c l}$.

Step 2: Select $n$ eigenvectors $v_{i} \in \mathbb{C}^{n(r+1)}$ of $\mathcal{N}$ associated with $\lambda_{i}(i=1, \cdots, n)$.

Step 3: Determine $X \in \mathbb{C}^{n \times n}$ and $Y \in \mathbb{C}^{r n \times n}$ such that $\left(\begin{array}{c}X \\ Y\end{array}\right)=\left(\begin{array}{c}v_{1} \cdots v_{n}\end{array}\right)$.

Step 4: If $\operatorname{det}(X) \neq 0$, then there exists a solution $K$ for the GARE (3), given by (6) leading to $A_{c l}$ with the desired eigenspectrum $\Lambda_{\text {desired }}$.

Remark 1 The proposed algorithm should be associated with some comments: 
- If a complex $\lambda_{i}$ is chosen, $\bar{\lambda}_{i}$ should be also considered and the pair $\left(v_{i}, \bar{v}_{i}\right)$ be selected, in order to allow real-valued solution $K$ (see [1]).

- If the eigenvalues $\lambda_{i} \in \Lambda_{\text {desired }}$ have a multiplicity greater than one, the matrix $\mathcal{N}$ could be non-diagonalizable and could imply proper invariant subspaces not restricted to eigenvectors. It is then possible to build the desired invariant subspace by considering the canonical Schur decomposition of $\mathcal{N}$ and reordering it, by extending the approach proposed in [24] (see for more details ([14,1])).

- A necessary condition for the invertibility of $X$ is that each of the eigenvalues $\lambda_{i} \in \Lambda_{\text {desired }}$ is an $\left(\mathcal{N}_{11}, \mathcal{N}_{12}\right)$ controllable eigenvalue. This is an extension of [1, Theorem 6.2.5] in the case of non trivial time preference rate. Contrary to the one-player case (see [23]), the above conditions are not sufficient.

Remark 2 It should be noticed that there exists a large panel of numerical methods to solve the GARE in the literature. Among them, we can emphasize the Newton-Raphson method and its refinements [7,15,9] and the homotopy continuation method [3]. Nevertheless, the Newton-Raphson iterative methods have not been chosen because they do not ensure a convergence to the solution with the desired closed-loop eigenspectrum $\Lambda_{\text {desired }}$ among all the possible (non-unique) solutions. The homotopy methods necessitate also to track the path of homotopy map over a finite horizon (by using a Newton-Raphson method at each increment or by discretizing the initial value problem related to the arc-length of the trajectory).

\section{Open-Loop Nash Strategy in Continuous-Time with Discount Rates}

Consider a $r$-player linear-quadratic differential game (see [8]), on a finite time horizon, defined by

$$
\dot{x}(t)=A x(t)+\sum_{i=1}^{r} B_{i} u_{i}(t), \quad x\left(t_{0}\right)=x_{0},
$$

where $x \in \mathbb{R}^{n}, u_{i} \in \mathcal{U}_{a d, i} \subset \mathbb{R}^{m_{i}}(i \in\{1 ; \cdots ; r\}) ; n, m_{i} \in \mathbb{N} ; \mathcal{U}_{a d, i}$ is the admissible set of the controls $u_{i}$ and with the cost functionals $\tilde{J}_{i}(i \in\{1 ; \cdots ; r\})$, including a time preference rate $\alpha_{i}$, given by

$$
\tilde{J}_{i}\left(x_{0}, u_{i}, u_{-i}\right)=\frac{1}{2} x_{f}^{T}\left(e^{\alpha_{i} t_{f}} K_{i f}\right) x_{f}+\frac{1}{2} \int_{t_{0}}^{t_{f}}\left(x^{T}\left(e^{\alpha_{i} t} Q_{i}\right) x+\sum_{j=1}^{r} u_{j}^{T}\left(e^{\alpha_{i} t} R_{i j}\right) u_{j}\right) \mathrm{d} \tau,
$$

where $x_{f}=x\left(t_{f}\right)$ and $u_{-i}=\left(u_{1} ; \cdots ; u_{i-1} ; u_{i+1} ; \cdots ; u_{r}\right)$ is a shortcut to denote the controls of all the players distinct from Player $i$. We note also $\mathcal{U}_{a d,-i}=\mathcal{U}_{a d, 1} \times \cdots \times \mathcal{U}_{a d, i-1} \times \mathcal{U}_{a d, i+1} \times \cdots \times \mathcal{U}_{a d, r}$. All weighting matrices are constant and symmetric with $Q_{i} \geq 0, K_{i f} \geq 0, R_{i j} \geq 0(i \neq j)$ and $R_{i i}>0$.

In order to define a Nash strategy, we introduce the rational reaction set $\mathcal{R}_{i}\left(u_{-i}\right)$ of the Player $i$ as $\left\{\tilde{u}_{i} ; \tilde{J}_{i}\left(x_{0}, \tilde{u}_{i}, u_{-i}\right) \leq\right.$ $\left.\tilde{J}_{i}\left(x_{0}, \tilde{u}_{i}^{*}, u_{-i}\right), \forall u_{i} \in \mathcal{U}_{a d, i}\right\}$.

Definition 1 A Nash strategy is a r-uplet $\left(u_{1}^{*} ; \cdots ; u_{r}^{*}\right)$ satisfying the following inclusions (see [28]):

$$
u_{i}^{*} \in \mathcal{R}_{i}\left(u_{-i}^{*}\right) ; \quad \forall i=1 ; \cdots ; r .
$$

By considering an open-loop information structure scheme (see $[17,33,8]$ ), the controls are only functions of time. The Pontryagin's Maximum Principle could be applied for each player, or inclusion (9). When the time preference rates $\alpha_{i}$ are not necessary null, we obtain the control laws proposed in [2]. These results are gathered in the following theorem.

Theorem 4 A Nash strategy $r-$ uplet $\left(u_{1}^{*} ; \cdots ; u_{r}^{*}\right)$ is given by

$$
u_{i}^{*}(t)=-R_{i i}^{-1} B_{i}^{T} \psi_{i}(t), \quad \forall i=1 ; \cdots ; r
$$

where the costate vectors $\psi_{i}(t)$, associated with the dynamic constraint (7) for the Player $i$, verify the necessary conditions, for each player $i,(\forall i=1 ; \cdots ; r)$ and the associated transversality conditions

$$
\dot{\psi}_{i}(t)=-Q_{i} x(t)-\left(A^{T}+\alpha_{i} I_{n}\right) \psi_{i}(t), \quad \psi_{i}\left(t_{f}\right)=K_{i f} x\left(t_{f}\right) .
$$


Proof Let us introduce the Hamiltonian $\mathcal{H}_{i}$ associated with each player $i$, with a temporary costate vector $p_{i}(t) \in \mathbb{R}^{n}$, related to the dynamic constraint (7):

$$
\mathcal{H}_{i}\left(t, x, p_{i}, u_{i}, u_{-i}\right)=p_{i}^{T}\left(A x+\sum_{j=1}^{r} B_{j} u_{j}\right)+\frac{1}{2}\left(x^{T}\left(e^{\alpha_{i} t} Q_{i}\right) x+\sum_{j=1}^{r} u_{j}^{T}\left(e^{\alpha_{i} t} R_{i j}\right) u_{j}\right) .
$$

Applying the Pontryaguin's Maximum Principle to each inclusion (9) leads to

$$
\begin{aligned}
\dot{p}_{i} & =-\frac{\mathrm{d} \mathcal{H}_{i}}{\mathrm{~d} x}\left(t, x, p_{i}, u_{i}^{*}, u_{-i}^{*}\right)=-e^{\alpha_{i} t} Q_{i} x-A^{T} p_{i} ; \\
0 & =\frac{\mathrm{d} \mathcal{H}_{i}}{\mathrm{~d} u_{i}}\left(t, x, p_{i}, u_{i}^{*}, u_{-i}^{*}\right)=e^{\alpha_{i} t} R_{i i} u_{i}^{*}+B_{i}^{T} p_{i} .
\end{aligned}
$$

The final state $x\left(t_{f}\right)$ being free, the transversality condition is given by:

$$
p_{i}\left(t_{f}\right)=\frac{\mathrm{d}}{\mathrm{d} x_{f}}\left(\frac{1}{2} x_{f}^{T} e^{\alpha_{i} t_{f}} K_{i f} x_{f}\right)=e^{\alpha_{i} t_{f}} K_{i f} x_{f} .
$$

Due to the presence of the exponential terms, time-varying coefficients occur in equations (13), (14) and (15). In order to obtain time invariant differential equations, we propose the following change of variable concerning the temporary costate vectors $p_{i}(t): \psi_{i}(t)=e^{-\alpha_{i} t} p_{i}(t)$.

The transversality condition (15) becomes (11). Moreover due to the invertibility assumption of $R_{i i}$, and the invertibility of the exponential scalar, the equation (14) implies $u_{i}^{*}(t)=-R_{i i}^{-1} B_{i}^{T} e^{-\alpha_{i} t} p_{i}(t)$, that is the relation (10).

To end the proof, the necessary condition (13) are reformulated thanks to the change of variable introducing $\psi_{i}(t)$ :

$$
\dot{\psi}_{i}=-\alpha_{i} \psi_{i}+e^{-\alpha_{i} t} \dot{p}_{i}=-\alpha_{i} \psi_{i}-Q_{i} x-A^{T} p_{i} e^{-\alpha_{i} t}=-Q_{i} x-\left(A^{T}+\alpha_{i} I_{n}\right) \psi_{i} .
$$

Differential equations (7) and (11) could be summarized into the extended linear system

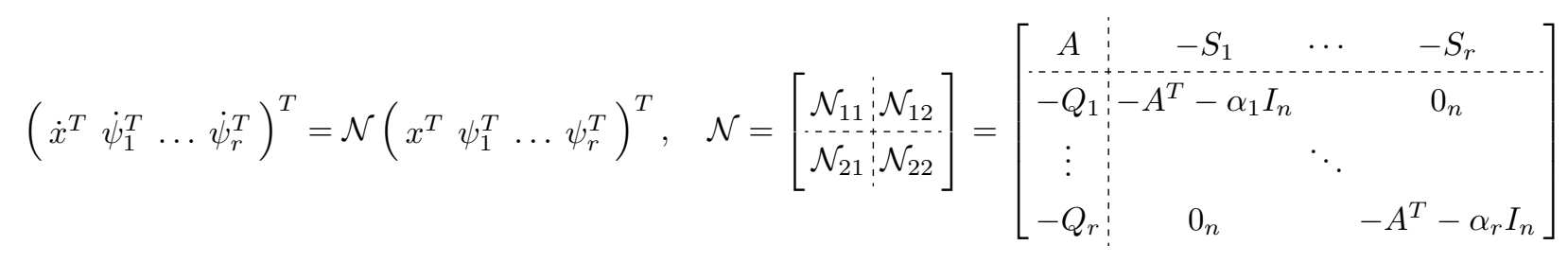

where the notation $S_{i}=B_{i} R_{i i}^{-1} B_{i}^{T}$ is used. The linear system (17) with the initial condition $x\left(t_{0}\right)=x_{0}$ and the transversality conditions (11) consists in a two-point boundary value problem. The following section provides a solution of such a problem via Coupled Differential or Algebraic Riccati Equations.

\section{Coupled Differential or Algebraic Riccati Equations}

The system (17) being linear, it is possible to set matrices $K_{i}(t)$ defined on $\left[t_{0} ; t_{f}\right]$ by $\psi_{i}(t)=K_{i}(t) x(t)$. The matrices $K_{i}(t)$ satisfy the following Coupled Differential Riccati Equations (CDRE) and the associated transversality condition, induced by equation (11):

$$
\dot{K}_{i}=-K_{i} A-Q_{i}-\left(A^{T}+\alpha_{i} I_{n}\right) K_{i}+K_{i} \sum_{j=1}^{r} S_{j} K_{j}, \quad K_{i f}=K_{i}\left(t_{f}\right) .
$$


The extended variable $K(t)=\left(K_{1}^{T}(t) \ldots K_{r}^{T}(t)\right)^{T}$ allows to rewrite the CDRE (18) in a compact form as a single GDRE $([1,14])$. This implies that the tools presented in section 2 can be used here. In the following section, this framework and these properties will be used in order to provide a non-uniform multiple pole shifting.

\section{Non-Uniform Pole Shifting in Continuous-Time}

Let us consider the multivariable system, with the pair $(A, B)$ controllable,

$$
\dot{x}=A x+B u
$$

We note $\check{n}_{\mathrm{r}}$ and $\check{n}_{\mathrm{c}}$ the numbers of real eigenvalues of $A$ and of the pairs of conjugate complex eigenvalues of $A$ $\left(\check{n}_{\mathrm{r}}+2 \check{n}_{\mathrm{c}}=n\right)$. The problem is to determine a real-valued gain feedback matrix $G$, i.e. $u(t)=G x(t)$, such that some or all eigenvalues of matrix $A$ are shifted $\left(n_{\mathrm{r}} \leq \check{n}_{\mathrm{r}}\right.$ real eigenvalues and $n_{\mathrm{c}} \leq \check{n}_{\mathrm{c}}$ conjugate pairs of eigenvalues) to predefined desired locations in the complex plane. The purpose is to shift all eigenvalues that need to be shifted in a single step. Thus non-uniform shifts are operated simultaneously. The whole desired closed-loop spectrum will be noted $\Lambda_{\text {desired }}$. Therefore, the approach examined here is quite different from the global shift proposed by [5], or iterative shifts proposed by [25]. We study a non-uniform shift technique of the eigenvalues, that is each modified eigenvalue or conjugate pair of eigenvalues has its own shift amplitude. This is for example the case in Figure 1, where $n_{\mathrm{r}}=\check{n}_{\mathrm{r}}=2$ and $n_{\mathrm{c}}=\check{n}_{\mathrm{c}}=1$ : the conjugate pair of poles are shifted by $s_{1}$, and the real eigenvalues are shifted respectively by $s_{2}$ and $s_{3}$. We consider the case where $s_{1}, s_{2}$ and $s_{3}$ are not necessarily equal as depicted on Figure 1.

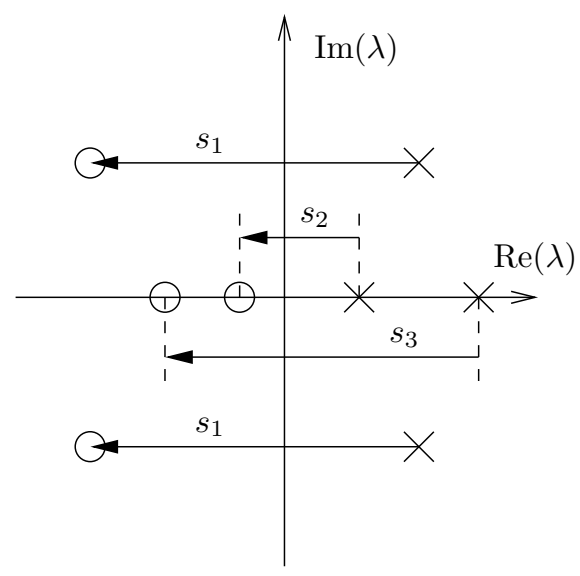

Fig. 1. Non-uniform poles shifting. $\times$ denotes the eigenvalues of $A$ and $\bigcirc$ denotes the closed-loop desired eigenvalues.

The main idea is to use the framework of CARE for a specific characteristic matrix $\mathcal{N}$ including the desired shifted eigenvalues in order to solve such a non-uniform pole shifting problem. The first step is to reformulate the problem as defined by the system (19) into a gametheoretic framework as given in (7). To formulate a new special Nash game, some fictitious players are introduced in the dynamic equation. We choose the number of fictitious players equal to $r=n_{\mathrm{r}}+n_{\mathrm{c}}$ (and not $r=n_{\mathrm{r}}+2 n_{\mathrm{c}}$, as it will be seen in Remark 3 ). In addition we impose

$$
B_{i}=B, \quad \forall i \in\{1 ; \cdots ; r\}, \quad u=\sum_{i=1}^{r} u_{i}
$$

The criteria $\tilde{J}_{i}(\forall i=1 ; \cdots ; r)$ are restricted to the following form: $J_{i}\left(x_{0}, u_{i}, u_{-i}\right)=\frac{1}{2} \int_{t_{0}}^{+\infty} u_{i}^{T}\left(e^{\alpha_{i} t} R_{i i}\right) u_{i} \mathrm{~d} \tau$.

The set of criteria $J_{i}$ could be interpreted as a compromise to reach, between the weighted input energies of each 
player. The characteristic matrix associated with an open-loop Nash game becomes

$$
\mathcal{N}=\left[\begin{array}{cccc}
A & -S_{1} & \cdots & -S_{r} \\
0_{n}-A^{T}-\alpha_{1} I_{n} & & 0_{n} \\
\vdots & & \ddots & \\
0_{n} & 0_{n} & & -A^{T}-\alpha_{r} I_{n}
\end{array}\right]
$$

Such a particular matrix $\mathcal{N}$ is upper triangular per blocks, with matrices $A$ or $\left(-A^{T}-\alpha_{i} I_{n}\right)$ on the diagonal. The time preference rates $\alpha_{i}$ should be designed to allow the inclusion of the desired closed-loop eigenvalues in the spectrum of $\mathcal{N}$. Let us define the sets $\mathcal{I}_{i}=\left\{j \in\{1, \cdots, n\} \mid \operatorname{Im}\left(\lambda_{\text {desired }, i}\right)=\operatorname{Im}\left(\lambda_{j}(A)\right)\right\}, \forall i \in\{1, \cdots, n\}$. The time preference rates $\alpha_{i}$ being real, it is only possible to reach a desired eigenvalue $\lambda_{\text {desired, } i}$ if there exists at least an eigenvalue of $A$ having the same imaginary part. The non-uniform desired shift is possible if $\mathcal{I}_{i} \neq \varnothing, \forall i \in\{1, \cdots, n\}$. Then $\alpha_{i}$ should be designed as $\alpha_{i}=-\operatorname{Re}\left(\lambda_{\mathrm{i}, \text { desired }}+\lambda_{j_{0}}(A)\right), j_{0} \in \mathcal{I}_{i}$, ensuring

$$
\Lambda_{\text {desired }} \subset \lambda(\mathcal{N})=\lambda(A) \bigcup\left(\bigcup_{i=1}^{r} \lambda\left(-A^{T}-\alpha_{i} I_{n}\right)\right)
$$

The number of possible choices of $\alpha_{i}$ depends on the cardinality of the set $\mathcal{I}_{i}$. Notice that the eigenvalues of $A$ are included in the spectrum of $\mathcal{N}$. This is useful for eigenvalues that do not need shifting and justifies that we select only $r \leq n$ fictitious players, instead of $n$. The choice of weighting matrices $R_{i i}$ and $\alpha_{i}$ allows a degree of freedom. When the matrices $R_{i i}(\forall i=1 ; \ldots ; r)$ are imposed, the whole number of choices for the time preference rates $\alpha_{i}$ is equal to $\left(\check{n}_{\mathrm{r}}\right)^{n_{\mathrm{r}}} \times \check{n}_{\mathrm{c}}$. The closed-loop eigenspectrum is not influenced by the choice of weighting matrices $R_{i i}$. Their selection is only guided by numerically ensuring that the matrix $X$ in the invariant subspace decomposition is invertible and thus allowing to provide a finite solution of the CARE associated with the characteristic matrix $\mathcal{N}$, with the desired eigenspectrum $\Lambda_{\text {desired }}$.

Remark 3 It is important to notice that for the pair $\left(\lambda_{\text {desired }, i} ; \bar{\lambda}_{\text {desired }, i}\right)$, the pair $\left(v_{i} ; \bar{v}_{i}\right)$ should be selected according to remark 1. Thus $v_{i}$ and $\bar{v}_{i}$ should be generated by the same block $-A^{T}-\alpha_{i} I_{n}$, which explains why $r=n_{r}+n_{c}$. If $r=n_{r}+2 n_{c}$, the eigenspace associated with $\bar{\lambda}_{i}$ is not restricted to a vector and it will be difficult to select precisely $\bar{v}_{i}$ as eigenvector of $\mathcal{N}$.

Remark 4 We present here a global methodology to design a non-uniform multiple pole shifting, in a single step. Nevertheless it is obviously possible to adapt our approach to provide an iterative scheme by considering only $r=1$ and applying successively our method to each real desired eigenvalue or each conjugate pair of desired complex eigenvalues. Such an iterative algorithm could be useful for large scale systems, that is with large $n$ and $r$. In such a case, the size of the matrix $\mathcal{N}$ could induce numerical problems making the use of the proposed single step approach more difficult.

The same idea can be adapted to the framework of discrete-time. That is the aim of the following sections.

\section{Open-Loop Nash Strategy in Discrete-Time}

In this section, we consider the framework of discrete-time for the game, that is the $r$-player difference game defined by:

$$
x(k+1)=A x(k)+\sum_{i=1}^{r} B_{i} u_{i}(k), \quad x\left(k_{0}\right)=x_{0},
$$

with the same notation of the section 3 and the cost function $\tilde{J}_{i}$ associated with each player $i \in\{1, \cdots, r\}$, including a time preference rate (in discrete-time) $\rho_{i}$, with a finite time horizon $\left[k_{0}, k_{f}\right]$, defined by

$$
\tilde{J}_{i}\left(x_{0}, u_{i}, u_{-i}\right)=\frac{1}{2} x\left(k_{f}\right)^{T} K_{i, f} x\left(k_{f}\right)+\frac{1}{2} \sum_{k=k_{0}}^{k_{f}-1}\left(x(k)^{T}\left(\rho_{i}^{k} Q_{i}\right) x(k)+\sum_{j=1}^{r} u_{j}(k)^{T}\left(\rho_{i}^{k} R_{i j}\right) u_{j}(k)\right) .
$$


The definition of the Nash strategy (definition 1) is still true in the discrete-time case, thus the necessary conditions for the Nash strategy are given by the following theorem via an extension of the result in [29].

Theorem 5 A Nash strategy r-uplet is given by

$$
u_{i}^{*}(k)=-R_{i i}^{-1} B_{i}^{T} \psi_{i}(k+1), \quad \forall i \in\{1, \cdots, r\},
$$

where the costate vectors $\psi_{i}(k)$ verify the necessary conditions

$$
\frac{1}{\rho_{i}} \psi_{i}(k)=Q_{i} x(k)+A^{T} \psi_{i}(k+1), \forall i \in\{1, \cdots, r\}
$$

and the transversality conditions $\psi_{i}\left(k_{f}\right)=K_{i, f} x\left(k_{f}\right)$. The conditions could be reformulated into an extended linear system

$$
\underbrace{\left[\begin{array}{cccc}
I_{n} & S_{1} & \cdots & S_{r} \\
0_{n} & A^{T} & & 0_{n} \\
\vdots & & \ddots & \\
0_{n} & 0_{n} & & A^{T}
\end{array}\right]}_{\mathcal{L}}\left(\begin{array}{c}
x(k+1) \\
\psi_{1}(k+1) \\
\vdots \\
\psi_{r}(k+1)
\end{array}\right)=\underbrace{\left[\begin{array}{cccc}
A & 0_{n} & \cdots & 0_{n} \\
-Q_{1} & \frac{1}{\rho_{1}} I_{n} & & 0_{n} \\
\vdots & & \ddots & \\
-Q_{r} & 0_{n} & & \frac{1}{\rho_{r}} I_{n}
\end{array}\right]}_{\mathcal{G}}\left(\begin{array}{c}
x(k) \\
\psi_{1}(k) \\
\vdots \\
\psi_{r}(k)
\end{array}\right) .
$$

Proof The proof of Theorem 5 follows the one of Theorem 4 by considering an Hamiltonian associated with each player $i$ :

$$
\mathcal{H}_{i}\left(k, x, p_{i}, u_{i}, u_{-i}\right)=p_{i}^{T}(k)\left(A x+\sum_{j=1}^{r} B_{j} u_{j}\right)+\frac{1}{2}\left(x^{T}\left(\rho_{i}^{k} Q_{i}\right) x+\sum_{j=1}^{r} u_{j}^{T}\left(\rho_{i}^{k} R_{i j}\right) u_{j}\right)
$$

and by applying the change of variable $\psi_{i}(k)=\frac{p_{i}(k)}{\rho_{i}^{k-1}}$.

As in the continuous-time case, we can search in the form $\psi_{i}(k)=K_{i}(k) x(k)$. By denoting $\phi(k)=\left(I_{n}+\sum_{j=1}^{r} S_{i} K_{i}(k)\right)$, we have the CARE in discrete-time $\forall i \in\{1, \cdots, r\}$ :

$$
\frac{1}{\rho_{i}} K_{i}(k)=Q_{i}+A^{T} K_{i}(k+1)(\phi(k+1))^{-1} A
$$

and the closed-loop dynamic is defined by $x(k+1)=(\phi(k+1))^{-1} A x(k)$.

When the time horizon is infinite, the CARE (29) become algebraic, that is $K_{i}$ are independent on the time $k$. Such a solution verifies the following theorem

Theorem 6 [1] Let us consider $\mathcal{L}$ and $\mathcal{G}$ defined by (27). If $A$ is invertible, then the matrix $\mathcal{L}$ is also invertible. This implies that the solutions $K_{i}$ are obtained by the invariant subspaces of the matrix $\mathcal{L}^{-1} \mathcal{G}$, which spectrum contains the spectrum of $\phi^{-1} A$.

Proof The property is verified straightforwardly due to the relation

$$
\mathcal{L}\left[\begin{array}{llll}
I_{n} & K_{1}^{T} & \ldots & K_{r}^{T}
\end{array}\right]^{T} \phi^{-1} A=\mathcal{G}\left[\begin{array}{llll}
I_{n} & K_{1}^{T} & \ldots & K_{r}^{T}
\end{array}\right]^{T} .
$$

Simultaneously the relation (30) allows to obtain solutions of (29) via the invariant subspaces of the matrix $\mathcal{N}=$ $\mathcal{L}^{-1} \mathcal{G}$. By using the relation $(29)$, the closed loop is given by $\mathcal{N}_{11}+\mathcal{N}_{12} K=\phi^{-1} A$.

Remark 7 If the matrix A is not invertible, the result of theorem (6) can be extended in the framework of deflating subspaces of the pencil $(\mathcal{L}, \mathcal{G})$ (see [22]). Notice also that the controls $u_{i}^{*}$ defined by (25) can be rewritten as $u_{i}^{*}=$ $-R_{i i}^{-1} B_{i}^{T} K_{i} \phi^{-1} A x(k)$. 


\section{Pole Homothety}

Let us consider in this section, the multivariable system, with the pair $(A, B)$ controllable: $x(k+1)=A x(k)+B u(k)$. As defined in the continuous-time part of the paper, $\check{n}_{r}$ and $\check{n}_{c}$ are the numbers of real eigenvalues of $A$ and of the pairs of conjugate complex eigenvalues of $A$. The problem is to determine a real-valued gain feedback $G, u(k)=G x(k)$ such that some or all eigenvalues of matrix $A$ are homothetically modified $\left(n_{r} \leq \check{n}_{r}\right.$ and $\left.n_{c} \leq \check{n}_{c}\right)$ with a rate $h_{i} \in \mathbb{R}$ to obtained a predefined desired locus in the complex plane, such for instance in Fig. 4.

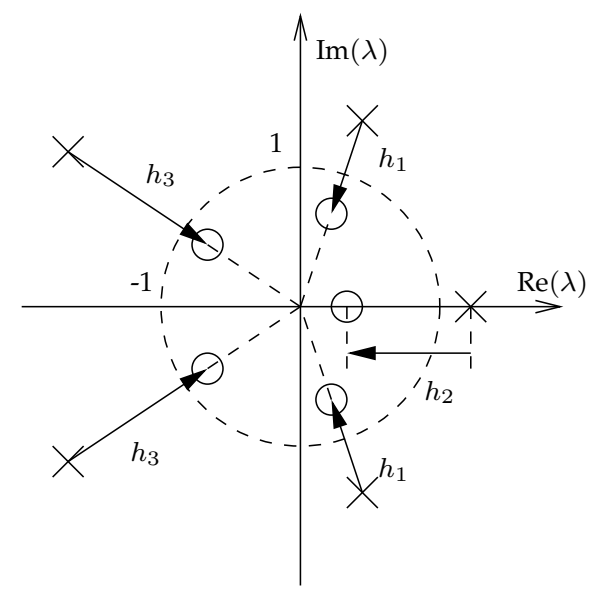

Fig. 2. Eigenvalues of matrix $A$ and closed-loop homothetic ones (with homothetic rates $h_{i}$ ). $\times$ denote eigenvalues of $A$ and the closed-loop ones.

In order to solve such a problem, as in the continuous time, we consider a fictitious Nash game involving $r=n_{\mathrm{r}}+n_{\mathrm{c}}$ players defined by the relation $(20)$. The criteria $\tilde{J}_{i}$ are restricted to the case $J_{i}\left(x_{0}, u_{i}, u_{-1}\right)=\sum_{k=0}^{+\infty} u_{i}^{T}\left(\rho_{i}^{k} R_{i i}\right) u_{i}$ such that

$$
\mathcal{L}^{-1} \mathcal{G}=\left[\begin{array}{c:ccc}
A & -\frac{S_{1} A^{-T}}{\rho_{1}} & \cdots & -\frac{S_{r} A^{-T}}{\rho_{r}} \\
\hdashline 0_{n} & \frac{A^{-T^{2}}}{\rho_{1}} & & 0_{n} \\
\vdots & & \ddots & \\
0_{n} & 0_{n} & & \frac{A^{-T}}{\rho_{r}}
\end{array}\right] .
$$

The methodology of selecting $\rho_{i}$ is the same as in continuous time. Let us introduce, $\forall i \in\{1, \cdots, n\}$, the sets $\mathcal{J}_{i}=\left\{j \in\{1, \cdots, n\} \mid \operatorname{Arg}\left(\lambda_{\text {desired }, i}\right)=\operatorname{Arg}\left(\lambda_{j}(A)\right)\right\}$. The homothety is possible if $\mathcal{J}_{i} \neq \varnothing, \forall i \in\{1, \cdots, n\}$ and thus the value of $\rho_{i}$ should verify $\rho_{i}=\left\|\lambda_{j_{0}}\right\|\left\|\lambda_{\text {desired }, i}\right\|, j_{0} \in \mathcal{J}_{i}$.

\section{Numerical Example in continuous time}

In order to illustrate our methodology, we present the following example, with $n=4$ from Example 6.6.9 in [1] in continuous time:

$$
A=\left[\begin{array}{rrrr}
-0.0366 & 0.0271 & 0.0188 & -0.4555 \\
0.0482 & -1.0100 & 0.0024 & -4.0208 \\
0.1002 & 0.2855 & -0.707 & 1.329 \\
0 & 0 & 1 & 0
\end{array}\right] ; \quad B=\left[\begin{array}{rr}
0.4422 & 0.1761 \\
3.0447 & -7.5922 \\
-5.52 & 4.99 \\
0 & 0
\end{array}\right]
$$

The eigenvalues of $A$ are $0.2823+0.0853 i, 0.2823-0.0853 i,-0.3359$, and -1.9823 . That is $\bar{n}_{\mathrm{c}}=1$ and $\bar{n}_{\mathrm{r}}=2$. We want to maintain the real eigenvalue -1.9823 and shift the others in order to have the closed-loop eigenvalues 
$\Lambda_{\text {desired }}=\{-1+0.0853 i ;-1-0.0853 i ;-3 ;-1.9823\}$. It implies we have $n_{\mathrm{r}}=1 ; n_{\mathrm{c}}=1$ and $r=2$. We choose arbitrarly positive definite matrices $R_{11}$ and $R_{22}$, by avoiding equal matrices $R_{11}=I_{2}$ and $R_{22}=\operatorname{diag}(1 ; 2)$. Equal matrices $R_{i i}$ lead to equal matrices $S_{i i}$ and thus could generate dependent eigenvectors of $\mathcal{N}$. Among the several possibilities, we select the time preference rate as follows $\alpha_{1}=-(0.2823+(-1))=0.7177$, in order to shift the conjugate pair of eigenvalues and $\alpha_{2}=-(-1.9823+(-3))=4.9823$, in order to shift the desired real eigenvalue which is not an eigenvalue of $A$. By selecting the desired closed-loop eigenvalues and their eigenvectors associated with matrix $\mathcal{N}$, it leads to a complex matrices $X$ and $Y$ (not precised here for more convenience), but to real matrices $K_{1}$ and $K_{2}$ as expected $K_{1}=\left[\begin{array}{rrrr}-0.0465 & 0.0034 & -0.0007 & -0.0064 \\ 0.0552 & 0.0045 & -0.0006 & -0.0307 \\ 0.3863 & 0.0234 & -0.0031 & -0.1783 \\ 0.5470 & 0.0270 & -0.0034 & -0.2253\end{array}\right] ; K_{2}=\left[\begin{array}{rrrr}0.0259 & 0.0011 & -0.0037 & -0.0170 \\ 0.1712 & 0.0073 & -0.0245 & -0.1124 \\ -0.5855 & -0.0251 & 0.0838 & 0.3845 \\ 0.7458 & 0.0320 & -0.1068 & -0.4898\end{array}\right]$.

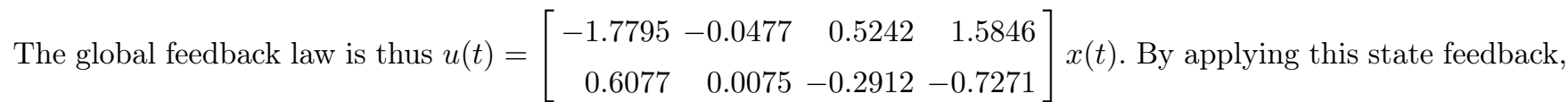
the resulting closed-loop system has the expected spectrum. The eigenvalues of $A$ and the closed-loop ones are depicted in the complex plan on Figure 3 . As precised above, with imposed $R_{i i}$, there is $\left(\check{n}_{\mathrm{r}}\right)^{n_{\mathrm{r}}} \times \check{n}_{\mathrm{c}}=2^{1} \times 1=2$

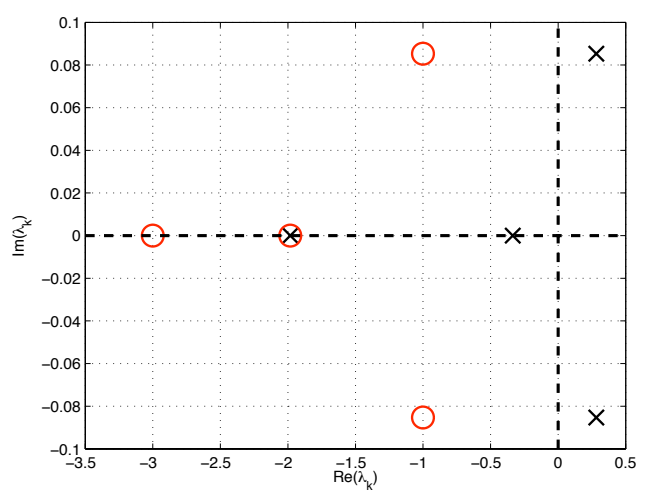

Fig. 3. Eigenvalues of matrix $A$ and closed-loop shifted ones. $\times$ denote eigenvalues of $A$ and $\bigcirc$ the closed-loop ones.

eventual solutions, the second solution corresponds to $\alpha_{1}=0.7177$ as previously and $\alpha_{2}=3.3359$, then $X$ is also invertible and we obtain: $K_{1}=\left[\begin{array}{rrrr}-0.0286 & 0.0042 & -0.0032 & -0.0182 \\ 0.0527 & 0.0044 & -0.0003 & -0.0291 \\ 0.3510 & 0.0219 & 0.0019 & -0.1551 \\ 0.4838 & 0.0243 & 0.0057 & -0.1838\end{array}\right] ; K_{2}=\left[\begin{array}{rrrr}0.3823 & 0.0164 & -0.0548 & -0.2511 \\ -0.3891 & -0.0167 & 0.0557 & 0.2555 \\ -0.9549 & -0.0410 & 0.1367 & 0.6272 \\ -0.3606 & -0.0155 & 0.0516 & 0.2368\end{array}\right]$.

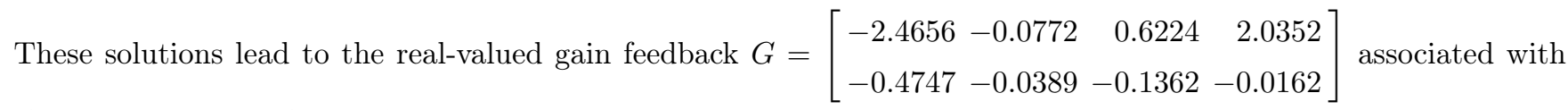
the same spectrum depicted on Figure 3.

\section{Illustration in discrete-time}

Let us now consider an example in discrete time, with $A=\left[\begin{array}{rrr}1.57 & 1.23 & 1.30 \\ 0.07 & 1.23 & -0.20 \\ -0.27 & -0.43 & 0.80\end{array}\right] ; B=\left[\begin{array}{r}0.6 \\ 0.1 \\ 1\end{array}\right]$. The eigenvalues of $A$ are $\{1.5 ; 1.05+0.31 i ; 1.05-0.31 i\}$, that is $\bar{n}_{\mathrm{c}}=1$ and $\bar{n}_{\mathrm{r}}=1$. We want to apply a non-uniform homothety to obtain a real eigenvalue with a modulus equal to 0.9 and a pair of complex conjugate eigenvalues with a modulus 
equal to 0.4 . We have thus $n_{\mathrm{r}}=1 ; n_{\mathrm{c}}=1$ and $r=2$. We choose arbitrarly positive definite matrices $R_{11}=2$ and $R_{22}=1$. Such we obtain $K_{1}=\left[\begin{array}{rrr}11.62 & 31.20 & 2.26 \\ 0.37 & -0.30 & 2.23 \\ 11.99 & 30.90 & 4.49\end{array}\right] ; K_{2}=\left[\begin{array}{lll}0.20 & 0.75 & -0.05 \\ 0.61 & 2.24 & -0.14 \\ 0.20 & 0.75 & -0.05\end{array}\right]$, which lead to the closed-loop spectrum as desired on Figure 4 and to the feedback gain including $G=\left[\begin{array}{lll}-1.32-3.46 & -0.80\end{array}\right]$.

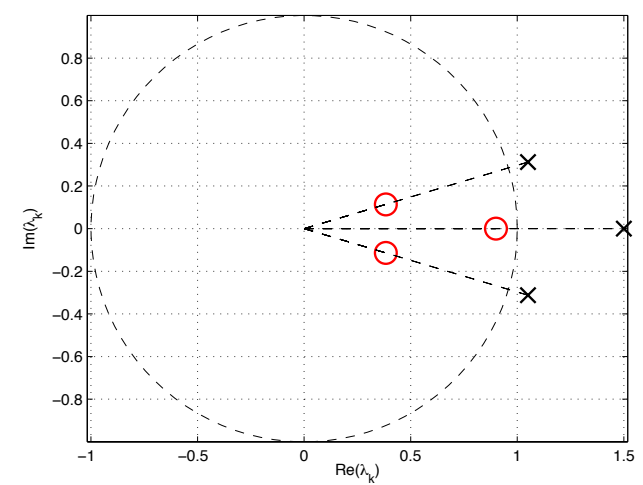

Fig. 4. Eigenvalues of matrix $A$ and closed-loop homothetic ones. $\times$ denote eigenvalues of $A$ and $\bigcirc$ the closed-loop ones.

\section{Conclusion}

The problem of pole shifting and of pole homothety in multivariable linear systems is revisited in a gametheoretic framework. It is shown that when a special Nash differential game is defined multiple non-uniform pole shifting can be obtained in a direct and simple way. The main idea is to introduce the desired eigenvalues of the closed loop system in the characteristic matrix associated with the non symmetric coupled Riccati equations by modifying the cost functionals in the special Nash game. Then, by an appropriate choice of the invariant subspace defining the solution of such matrix equations, a constant feedback gain matrix could be easily determined.

\section{Acknowledgements}

This work was partially supported by the project PICS CNRS n ${ }^{\circ} 5284$, by project CAPES/COFECUB n ${ }^{\circ} 701 / 11$, by ANR project ArHyCo, Programme "Systèmes Embarqués et Grandes Infrastructures" - ARPEGE, contract number ANR-2008 SEGI 004 01-30011459. E. B. Castelan and E. R. De Pieri have a financial support by CNPq Brazil.

\section{References}

[1] H. Abou-Kandil, G. Freiling, V. Ionescu, and G. Jank. Matrix Riccati Equations in Control and Systems Theory. Birkhäuser, 2003.

[2] H. Abou-Kandil and G. Jank. Open-loop Nash Riccati equations in games with discount rate. In Proceedings of the IFAC Conference on Control Applications of Optimization, Hungary, 2003.

[3] E. L. Allgower and K. Georg. Numerical Continuation Methods: An Introduction, volume 13 of Series in Computational Mathematics. Springer Verlag, Berlin, Heidelberg, New York, 1990.

[4] M. H. Amin. Optimal pole shifting for continuous multivariable linear systems. International Journal of Control, 41(3):701-707, 1985.

[5] B.D.O. Anderson and J. B. Moore. Optimal control: linear quadratic methods. Prentice-Hall International Editions, 1989.

[6] A.-R. S. Arar and M. E. Sawan. Optimal pole shifting for discrete time systems. In Proceedings of the American Control Conference, pages 1119-1120, 1992.

[7] T. P. Azevedo-Perdicoúlis and G. Jank. Iterative solution of algebraic matrix Riccati equations in open-loop Nash games. International Journal of Robust and Nonlinear Control, 15(2):55-62, 2004.

[8] T. Başar and G. J. Olsder. Dynamic Noncooperative Game Theory. SIAM, 1995.

[9] D. A. Bini, B. Iannazzo, and F. Poloni. A fast Newton's method for a nonsymmetric algebraic Riccati equation. SIAM J. Matrix Anal. Es Appl., 30(1):276-290, 2008. 
[10] H. Bourlès, Y. Joannic, and O. Mercier. $\rho$-stability and robustness: discrete-time case. International Journal of Control, 52(5):12171239,1990

[11] M. Chilali, P. Gahinet, and P. Apkarian. Robust pole placement in lmi regions. IEEE Transactions on Automatic Control, AC44(12):2257-2269, december 1999.

[12] E. Dockner, S. Jørgensen, N. Van Long, and G. Sorger. Differential games in economics and management science. Cambridge University Press, 2000.

[13] J. C. Engwerda. LQ Dynamic Optimization and Differential Games. Wiley, 2005.

[14] G. Freiling. A survey of nonsymmetric Riccati equations. Linear Algebra and its Applications, 351-352:243-270, 2002.

[15] C. H. Guo. A new class of nonsymmetric algebraic Riccati equations. Linear Algebra and its Applications, 426:636-649, 2007.

[16] M. Heymann. The pole shifting theorem revisited. IEEE Transactions on Automatic Control, 24(3):479-480, June 1979.

[17] Y. C. Ho. Survey paper: Differential games, dynamic optimization and generalized control theory. Journal of Optimization Theory and Applications, 6(3):179-209, 1970.

[18] J.-W. Jang, A. Lee, N. Bedrossian, and P. Spanos. Design of robust Nash game theoretic controllers with time domain constraints. In Proceedings of the American Control Conference, pages 5363-5368, Denver, Colorado, June 4-6 2003.

[19] M. Jungers. Discrete-time riccati equations in open-loop stackelberg games with time preference rates. In 3rd IFAC Symposium on System Structure and Control (SSSC). Foz do Iguassu, October 2007.

[20] M. Jungers. On Stackelberg linear quadratic games with time preference rate. IEEE Transactions on Automatic Control, 53(2):621625, March 2008.

[21] M. Jungers, H. Abou-Kandil, E. B. Castelan, and E. R. De Pieri. A Nash strategy approach for non-uniform multiple pole shifting. In Proceedings of the IFAC World Congress, Milan, Italia, 2011.

[22] M. Jungers, C. Oară, H. Abou-Kandil, and R. Ştefan. General matrix pencil techniques for solving non-symmetric algebraic riccati equations. SIAM, J. Matrix Anal. \&S Appl., 31(3):1257-1278, 2009.

[23] V. Kučera. A contribution to matrix quadratic equations. IEEE Transactions on Automatic Control, pages 344-347, June 1972.

[24] A. J. Laub. A Schur method for solving the algebraic riccati equation. IEEE Transactions on Automatic Control, 30:97-108, 1979.

[25] J. Medanic, H.S. Tharp, and W.R. Perkins. Pole placement by performance criterion modification. IEEE Transactions on Automatic Control, vol 33(5):469-472, may 1988.

[26] B. P. Molinari. The time-invariant linear-quadratic optimal control problem. Automatica, 13(4):347-357, July 1977.

[27] Y. Mori and E. Shimemura. On a shift of eigenvalues of a matrix by using a solution of an algebraic Riccati equation. International Journal of Control, 32(1):73-80, 1980.

[28] J.F. Nash. Equilibrium points in N-person games. Proceedings Nat. Acad. Science U.S., 36:48-49, 1950.

[29] R.S. Pindyck. Optimal economic stabilization policies under decentralized control and conflicting objectives. IEEE Transactions on Automatic Control, 22:517-530, 1977.

[30] J. Radon. Über die oszillationstheoreme der kunjugierten punkte beim probleme von lagrange. (german). Münchener Sitzungsberichte, 57:243-257, 1927.

[31] J. Rissanen. Control system synthesis by analogue computer based on the 'generalized linear feedback' concept. In Int. Sem. on Analog Computation to the Study of Chemical Processes, Brussels, 1960.

[32] P. K. Sharma. On pole assignment problem in polynomial rings. Systems \& Control Letters, 5(1):49-54, October 1984.

[33] M. Simaan and J. B. Cruz. On the solution of the open-loop Nash Riccati equations in linear quadratic differential games. International Journal of Control, 18(1):57-63, 1973.

[34] W. M. Wonham. On pole assignment in multi-input contrallable linear systems. IEEE Transactions on Automatic Control, 12:660665, 1967. 